\title{
The utility of MLPA in Familial Hypercholesterolemia diagnosis
}

\author{
George Valeriu Moldovan, Adina Huțanu², Liliana Demian³, Laszlo Hadadi, Bogdan Mănescu², \\ Minodora Dobreanu² \\ 1. Microbiology Department, George Emil Palade University of Medicine, Pharmacy, Science, and Technology of Targu Mures, Romania \\ 2. Laboratory Medicine Department, George Emil Palade University of Medicine, Pharmacy, Science, and Technology of Targu Mures, Romania \\ 3. Center for Advanced Medical and Pharmaceutical Research, George Emil Palade University of Medicine, Pharmacy, Science, and Technology of Targu \\ Mures, Romania \\ 4. Emergency Institute for Cardiovascular Diseases and Transplantation Targu Mures, Romania
}

Background: Familial Hypercholesterolemia $(\mathrm{FH})$ is an inherited disease, associated with an increased risk of atherosclerosis, manifested clinically as premature coronary heart disease. FH is biochemically characterized by increased Cholesterol and Low-density Lipoprotein Cholesterol serum levels. The diagnosis is often made using clinical scores however, the definitive FH diagnosis should point out the underlying molecular change, which can be: a point mutation within the three major genes, a number of single nucleotide polymorphisms determining the polygenic etiology, or copy number variations in the Low-density lipoprotein receptor gene. Objective: In the present study we investigated copy number variations as a possible etiological factor for $\mathrm{FH}$ in a cohort of patients with documented premature coronary heart disease. Methods: The study population consisted of 150 patients with premature coronary heart disease documented by angiography, all being under lipid-lowering therapy, and 20 apparently healthy controls. Serum lipids were assessed using the Cobas Integra 400 plus and commercial reagents. Copy number variations were evaluated with the SALSA MLPA Probemix P062 LDLR kit. Results: Cholesterol, Triglycerides, Lowdensity Lipoprotein Cholesterol and High-density Lipoprotein Cholesterol showed no difference between patients and controls. No copy number variations were detected in the investigated regions, namely all 18 exons and the promoter region of the Low-density lipoprotein receptor gene. Conclusions: Even in the presence of negative results, the Familial Hypercholesterolemia genetic diagnosis has to be further pursued in the presence of a clinical diagnosis, as the identification of the molecular etiology may bring additional clinical and therapeutical benefits, as well as open the possibility for "cascade screening".

Keywords: familial hypercholesterolemia, atherosclerosis, premature coronary heart disease, low-density lipoprotein receptor

Received 15 June 2021 / Accepted 21 July 2021

\section{Introduction}

Familial Hypercholesterolemia (FH) is an inherited disease, associated with an increased risk of atherosclerosis, manifested clinically as coronary heart disease (CHD) or stroke. From a biochemical point of view, $\mathrm{FH}$ is characterized by increased Cholesterol (Chol) and Low-density Lipoprotein Cholesterol (LDL-Chol) serum levels, with patients displaying normal Triglycerides (Trig) levels [1]. The main consequence of $\mathrm{FH}$ is the occurrence of premature $\mathrm{CHD}$, with a third up to one-half of untreated patients suffering a potentially lethal cardiac atherosclerotic event below the age of 55 for men and 60 in the case of women $[2,3]$.

The diagnosis is often made using clinical scores, such as the Simon Broome, Dutch Lipid Clinic Network (DLCN) or Make Early Diagnosis to Prevent Early Death (MEDPED), that rely on clinical and biochemical findings [4]. However, since it's primarily a genetic condition, the definitive FH diagnosis should point out the underlying molecular change [4] which can be: a) a point mutation within the three major genes (accounting for up to $80 \%$ of all genetically diagnosed $\mathrm{FH}$ cases [5]): Low-density lipoprotein receptor (LDLR) [6], Apolipoprotein B

* Correspondence to: George Valeriu Moldovan

E-mail: valeriu.moldovan@umfst.ro
(APOB), and Proprotein convertase subtilisin/kexin type 9 (PCSK9), b) a number of single nucleotide polymorphisms determining the polygenic etiology (in approximately 20\% [5] of FH cases, with point mutations in genes such as the LDL-receptor adaptor protein 1, Apolipoprotein E, Patatin-like phospholipase-domain-containing family, Lysosomal acid lipase, Signal-transducing adaptor protein family 1 [4], Cadherin EGF LAG seven-pass G-type receptor 2 and ATP binding cassette subfamily $G$ member 8 ), or c) copy number variations (CNV) (determining $\mathrm{FH}$ in $0-10 \%$ of investigated cases) [4].

In trying to identify a genetic FH cause, the LDLR gene is primarily studied by numerous investigators, given the fact that it harbors a large number of identified variants, many of which are considered pathogenic [6]. Copy number variations in this gene are described to be involved in FH etiology, but their frequency differs in various populations, as reported by several authors [4]. Multiplex ligationdependent probe amplification (MLPA) is considered to be the "gold standard" [7] technique in identifying small deletions and duplications, known as CNVs. One advantage of MLPA is that numerous available kits analyze CNVs' occurrence in different pathologies, such as the SALSA MLPA Probemix P062 LDLR, an in vitro diagnostic [8] tool able to investigate CNVs in all 18 exons of LDLR, as well as its promoter region. 
The present study aimed to investigate whether copy number variations were an etiological factor for $\mathrm{FH}$ in a cohort of patients with documented premature $\mathrm{CHD}$ and to compare the frequency of the identified structural aberrations with data from other populations since there is no incidence reported in the investigated population.

\section{Methods}

The study population consisted of 150 patients and 20 controls, all above 18 years of age, coming from the Central part of Romania. Enrollment criteria for the patients were: a) coronary heart disease documented by angiography, b) CHD diagnosis $\leq 55$ years old for men, $\leq 60$ years old for women. For the controls, the inclusion criteria consisted of: a) lack of any documented cardiovascular disease, b) no lipid-lowering therapy, c) LDL-Chol within the reference range, d) age between 18-55 years old. Exclusion criteria for the whole group were the presence of any metabolic disease that could be related to lipid modifications, such as chronic thyroid, kidney, or liver disorders, as well as diabetes.

All participants in the present study signed informed consent prior to their enrollment. The methodology was approved by the Ethics Committee of the George Emil Palade University of Medicine, Pharmacy, Science, and Technology of Tîrgu Mureș and complied with the World Medical Association's Declaration of Helsinki.

Since patients had a prior CHD diagnosis, they were all following lipid-lowering therapy, as prescribed by their consulting cardiologist.

Two blood samples were taken from each person included in this study. One was collected in a vacuum tube containing a separating gel to investigate serum lipids. Isolation of genomic DNA was performed from the leukocytes of peripheral blood collected in a vacuum tube containing ethylenediaminetetraacetic acid, using the GeneJET Genomic DNA Purification Kit (Thermo Fisher Scientific, Waltham, USA), as indicated by the manufacturer.

Serum lipids, namely Chol, Trig, LDL-Chol, and HDL-Chol were assessed with the Cobas Integra 400 plus (Hoffmann-La Roche, Switzerland) system, using reagents validated for clinical diagnosis, according to the products' inserts.
The SALSA MLPA Probemix P062 LDLR kit (MRC Holland, Amsterdam, the Netherlands) was used to analyze copy number variations. Samples were prepared according to the manufacture's protocol, using a Mastercycler Nexus GSX1 PCR system (Eppendorf, Hamburg, Germany) for amplification and a 3500xL Dx Genetic Analyzer system (Applied Biosystems, Foster City, California, United States) for fragment analysis while Coffalyser.NET software (version v.140721,1958, MRC Holland, Amsterdam, the Netherlands) allowed the interpretation of the results, considering a reference range between 0.7-1.3 copies.

MedCalc software [9] was used to compare the difference between the observed means in the two groups. A p-value $<0.05$ was considered statistically significant.

\section{Results}

Serum lipids within the two studied lots are shown in Table I. The MLPA analysis in the patients and control groups displayed no modifications regarding copy number variations within the investigated regions of LDLR. The electrophoretic pattern of a negative patient's sample is illustrated in Figure 1 (A and B).

\section{Discussion}

$\mathrm{FH}$ is often an underdiagnosed disease, displaying an estimated prevalence of 1:220 in various populations [3]. Although genetic testing has proven its utility in making the final diagnosis, it is not being widely used worldwide [10]. Additionally, even if molecular techniques are used, identification of the genetic cause in $\mathrm{FH}$ is often challenging, given the extensive number of point mutations within LDLR [4], as well as within the other genes involved, such as APOB, PCSK9, and Low-Density Lipoprotein Receptor Adaptor Protein 1 to name just the most significant. Added to these, the polygenic etiology and CNVs in the affected gene contribute to the complex genetic spectrum of $\mathrm{FH}$, although their incidence in the occurrence mechanism is lower [4]. These aspects may explain why a large proportion of patients may remain without an identifiable molecular cause, even in the presence of a diagnosis made according to the clinical scores [11].

In our study cohort, the patients' diagnosis was based on clinical findings, all being investigated by angiography

Table I. Serum lipid levels in the study cohort.

\begin{tabular}{|c|c|c|c|}
\hline & $\begin{array}{c}\text { Patients }(n=150) \\
\text { (men } n=120 ; \text { women } n=30)\end{array}$ & $\begin{array}{c}\text { Controls }(n=20) \\
\text { (men } n=12 ; \text { women } n=8)\end{array}$ & $p$-value \\
\hline $\begin{array}{c}\text { Cholesterol }(\mathrm{mg} / \mathrm{dl}) \\
\text { Men } \\
\text { Women } \\
\end{array}$ & $\begin{array}{c}170.9 \pm 51.2(73-460.7) \\
169.7 \pm 51.6(73-460.7) \\
175.6 \pm 50(92.6-300.21) \\
\end{array}$ & $\begin{array}{c}184.9 \pm 45.5(98.8-265.3) \\
180.4 \pm 38(98.8-233.6) \\
191.8 \pm 57(104.4-265.3)\end{array}$ & 0.24 \\
\hline $\begin{array}{c}\text { Triglycerides }(\mathrm{mg} / \mathrm{dl}) \\
\text { Men } \\
\text { Women }\end{array}$ & $\begin{array}{c}168.1 \pm 111.3(24.9-852.8) \\
175.8 \pm 118.9(56.9-852.8) \\
137.2 \pm 66.9(24.9-351.9)\end{array}$ & $\begin{array}{c}153.5 \pm 107.4(64.9-495.3) \\
157 \pm 83.2(64.9-317.4) \\
148.2 \pm 142.7(69-495.3)\end{array}$ & 0.58 \\
\hline $\begin{array}{c}\text { LDL-Cholesterol (mg/dl) } \\
\text { Men } \\
\text { Women }\end{array}$ & $\begin{array}{c}112.9 \pm 52.1(32.2-424.1) \\
111.7 \pm 52.1(32.2-424.1) \\
118 \pm 52.6(44.2-249.6)\end{array}$ & $\begin{array}{c}124.7 \pm 39.3(50.2-196.9) \\
123 \pm 34.3(50.2-168.2) \\
127.3 \pm 48.2(58.8-196.9)\end{array}$ & 0.33 \\
\hline $\begin{array}{c}\text { HDL-Cholesterol }(\mathrm{mg} / \mathrm{dl}) \\
\text { Men } \\
\text { Women }\end{array}$ & $\begin{array}{c}41.6 \pm 12.1(11.7-73.8) \\
39.9 \pm 11.3(11.7-70.24) \\
48.5 \pm 12.8(19.8-73.8)\end{array}$ & $\begin{array}{c}46.4 \pm 22.4(28.9-85) \\
41.5 \pm 10.4(28.9-59.6) \\
53.7 .4 \pm 18.2(33.8-85)\end{array}$ & 0.1 \\
\hline
\end{tabular}


A

ASR 73

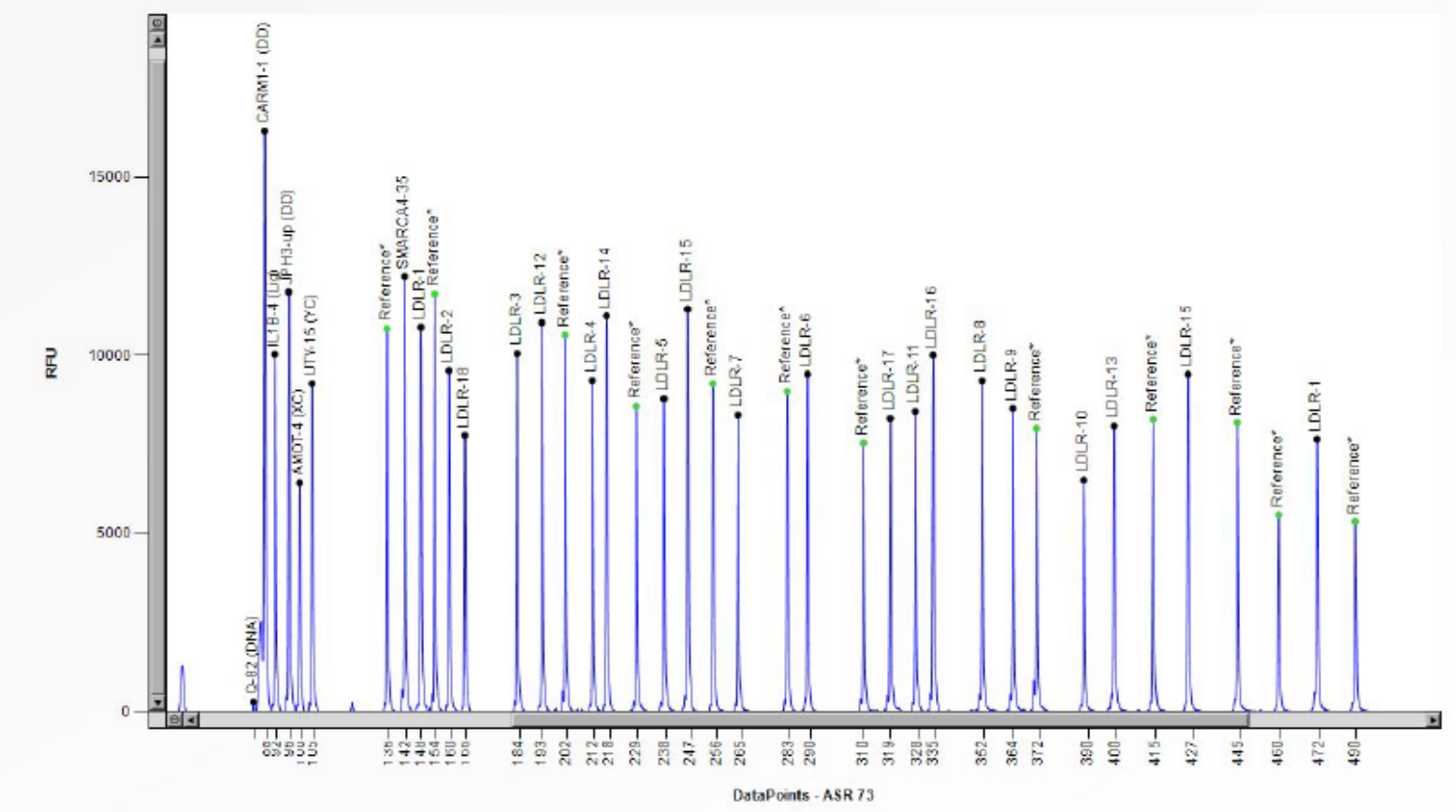

B

ASR 73

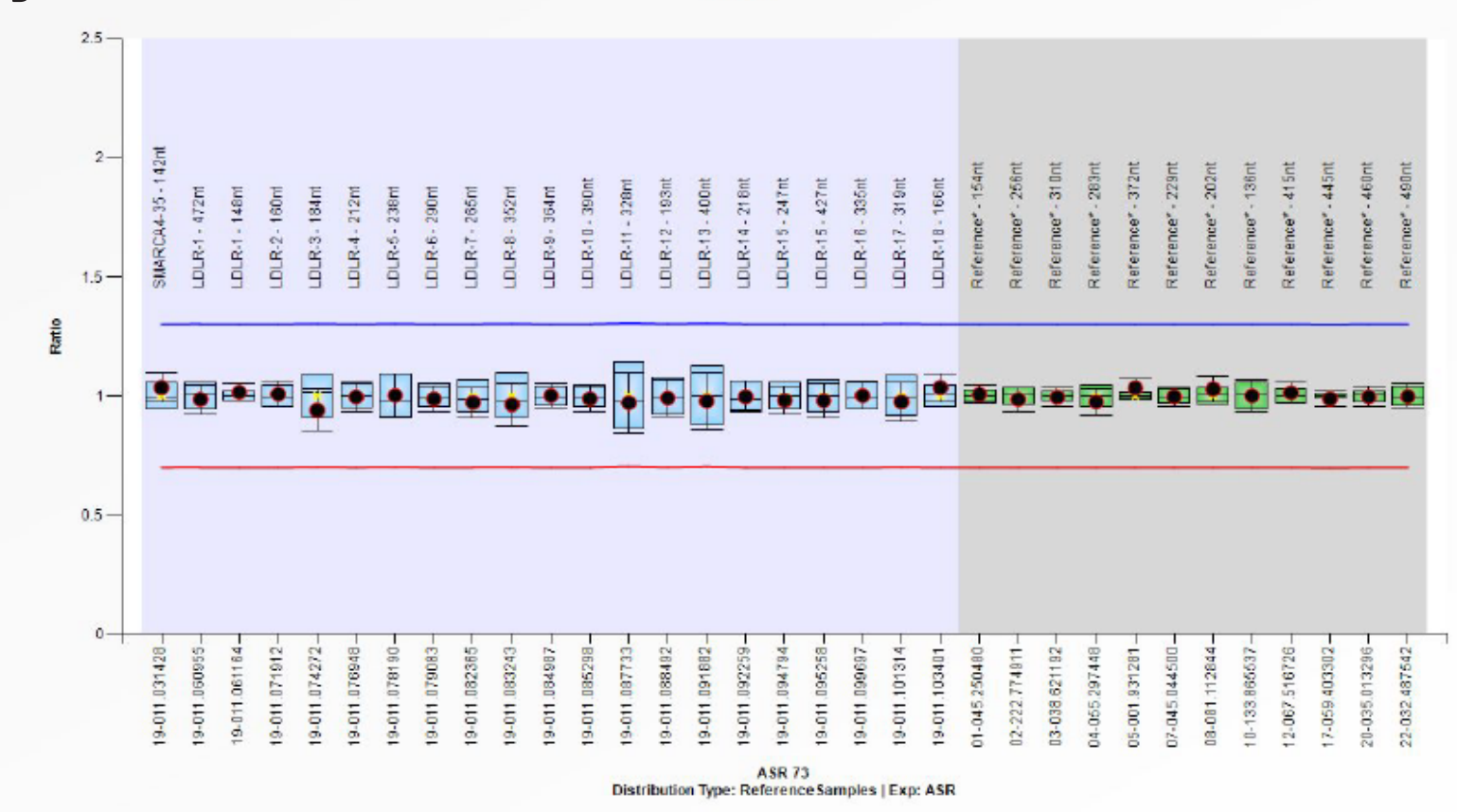

Fig. 1. A. Electrophoretic pattern of a negative sample investigated with SALSA MLPA Probemix P062 LDLR. Vertical axis: RFU-relative fluorescence units; horizontal axis: length (in base pairs) of the investigated fragment. B Electrophoretic pattern of a negative sample investigated with SALSA MLPA Probemix P062 LDLR. Vertical axis: Ratio, copy number reference range between 0.7-1.3; horizontal axis: chromosomal position of the investigated fragment.

secondary to a CHD event. Given the characteristics and inclusion criteria of our study group, serum lipid levels before the CHD diagnosis could not be evaluated. Since all patients were following lipid-lowering therapy when enrolled in the present study, it was to no surprise [12] that Chol, Trig, and LDL-Chol showed no statistical difference between patients and controls. Similarly, HDL-Chol, the main protector against atherosclerosis [13] was lower in patients, compared to controls. This was again in line with the results published by other authors since it has been recognized that statin therapy does not significantly increase HDL-Cholesterol serum levels [14].

MLPA is frequently regarded as the method of choice for the investigation of CNVs, because it has a superior 
resolution compared to other cytogenetic techniques, such as fluorescent in situ hybridization, array comparative genomic hybridization, or karyotyping [15]. On the other hand, new molecular genetic techniques, such as next-generation sequencing (NGS) [16] combined with bioinformatics have been used to investigate CNV's involvement in FH etiology [17], although the expense difference between the two approaches is rather significant, and NGS panels are still marked as "research use only" [18].

To date, copy number variations (manifested as loss-offunction deletions) associated with FH determinism were only described in the LDLR gene [4], and their prevalence is significantly lower, at least in comparison to FH caused by point mutations in the three major genes, LDLR, APOB, and PCSK9 [19]. The reduced incidence, as well as a probable uneven distribution between different populations, may be causing the disparate results published regarding CNVs in FH. Similar to our findings, other authors have failed to identify CNVs in LDLR, in the presence of a clinical diagnosis, even though the study of Pecin et al. described the presence of several point mutations in their studied population [20]. Contrariwise, other authors have reported the involvement of LDLR deletions in $\mathrm{FH}$ etiology in their study cohorts [21-23], albeit in a reduced number of subjects.

Our study has illustrated that copy number variations do not play an etiological role in FH determinism in our investigated population. Further testing, including screening for the most common point mutations in the three major genes, or even sequencing (Sanger or NGS) will be necessary to make a genetic diagnosis for these patients, even though they were all diagnosed based on clinical criteria, as well as being treated, according to the indications of their curing cardiologist, and current advice [24]. Finding the causal mutation remains a final goal, as it may allow for personalized medication [25] for these patients, as well as "cascade screening" [26] for their family members, in order to include them in CHD prevention programs.

\section{Conclusion}

Even in the presence of negative results, the Familial Hypercholesterolemia genetic diagnosis has to be further pursued in the presence of a clinical diagnosis. Although burdensome, identification of the molecular etiology will bring additional information to the affected patients, their families, as well as curing physicians.

\section{Authors' contribution}

VM - Conceptualization, Data curation, Investigation, Methodology, Validation, Writing - original draft, Writing - review \& editing

$\mathrm{AH}$ - Formal Analysis, Investigation, Methodology, Writing - review \& editing

LD - Formal Analysis, Investigation, Methodology, Resources, Writing - review \& editing

LH - Data curation, Methodology, Resources, Writing - original draft, Writing - review \& editing

BM - Formal Analysis, Resources, Software, Writing original draft, Writing - review \& editing

DM - Conceptualization, Methodology, Supervision, Validation, Writing - original draft, Writing - review \& editing.

\section{Conflict of interest}

None to declare.

\section{References}

1. Ogura M, Harada-Shiba M, Masuda D, Arai H, Bujo H, Ishibashi S, et al. Factors Associated with Carotid Atherosclerosis and Achilles Tendon Thickness in Japanese Patients with Familial Hypercholesterolemia: A Subanalysis of the Familial Hypercholesterolemia Expert Forum (FAME) Study. J Atheroscler Thromb. 2021;28:0-000. doi:10.5551/jat.62925.

2. Arnett DK, Blumenthal RS, Albert MA, Buroker AB, Goldberger ZD, Hahn EJ, et al. 2019 ACC/AHA Guideline on the Primary Prevention of Cardiovascular Disease: A Report of the American College of Cardiology/ American Heart Association Task Force on Clinical Practice Guidelines. Circulation. 2019;140:e596-646. doi:10.1161/CIR.0000000000000678.

3. Sturm AC, Knowles JW, Gidding SS, Ahmad ZS, Ahmed CD, Ballantyne $\mathrm{CM}$, et al. Clinical Genetic Testing for Familial Hypercholesterolemia: JACC Scientific Expert Panel. Journal of the American College of Cardiology. 2018;72:662-80.

4. Moldovan V, Banescu C, Dobreanu M. Molecular diagnosis methods in familial hypercholesterolemia. Anatolian Journal of Cardiology. 2020;23:120-7.

5. Trinder M, Li X, DeCastro ML, Cermakova L, Sadananda S, Jackson LM, et al. Risk of Premature Atherosclerotic Disease in Patients With Monogenic Versus Polygenic Familial Hypercholesterolemia. J Am Coll Cardiol. 2019;74:512-22.

6. The LDLR gene homepage - Global Variome shared LOVD. https:// databases.lovd.nl/shared/genes/LDLR. Accessed 13 Jun 2021.

7. MLPA: Multiplex Ligation-dependent Probe Amplification - MRC Holland. https://www.mrcholland.com/technology/mlpa. Accessed 1 Jun 2021.

8. MLPA $®$ MLPA $®$ MRC-Holland Product Description SALSA $®$ MLPA $®$ Probemix P062-D2 LDLR. www.mlpa.com. Accessed 3 Jun 2021.

9. MedCalc's Comparison of means calculator. https://www.medcalc.org/ calc/comparison_of_means.php. Accessed 2 Jun 2021.

10. Nordestgaard BG, Benn M. Genetic testing for familial hypercholesterolaemia is essential in individuals with high LDL cholesterol: Who does it in the world? European Heart Journal. 2017;38:1580-3. doi:10.1093/eurheartj/ehx136.

11. Nordestgaard BG, Chapman MJ, Humphries SE, Ginsberg HN, Masana L, Descamps OS, et al. Familial hypercholesterolaemia is underdiagnosed and undertreated in the general population: Guidance for clinicians to prevent coronary heart disease. Eur Heart J. 2013;34:3478-90. doi:10.1093/eurheartj/eht273.

12. Pang J, Chan DC, Watts GF. The Knowns and Unknowns of Contemporary Statin Therapy for Familial Hypercholesterolemia. Current Atherosclerosis Reports. 2020;22:1-10. doi:10.1007/s11883-020-00884-2.

13. Bandeali S, Farmer J. High-density lipoprotein and atherosclerosis: The role of antioxidant activity. Curr Atheroscler Rep. 2012;14:101-7. doi:10.1007/s11883-012-0235-2.

14. Woudberg NJ, Pedretti S, Lecour S, Schulz R, Vuilleumier N, James RW, et al. Pharmacological intervention to modulate HDL: What do we target? Frontiers in Pharmacology. 2018;8 JAN:989. doi:10.3389/ fphar.2017.00989.

15. Moldovan V, Moldovan E. Multiplex ligation-dependent probe amplification - a short overview. Rev Rom Med Lab. 2020;28:123-31.

16. lacocca M, Wang J, Dron J, Cao H, Robinson J, Mclntyre A, et al. DNA copy number variation screening in familial hypercholesterolemiarelated genes. Atherosclerosis. 2018;275:e79. doi:10.1016/j. atherosclerosis.2018.06.218.

17. lacocca MA, Wang J, Dron JS, Robinson JF, Mclntyre AD, Cao H, et al. Use of next-generation sequencing to detect LDLR gene copy number variation in familial hypercholesterolemia. J Lipid Res. 2017;58:2202-9. doi:10.1194/jlr.D079301.

18. CytoSure Comprehensive FH Panel. https://www.ogt.com/products/ product-search/cytosure-comprehensive-fh-panel/. Accessed 4 Jun 2021. 
19. Scholarship W, lacocca M, Hegele S, Program in Biochemistry G. Improving the genetic diagnosis of familial hypercholesterolemia. 2019. https://ir.lib.uwo.ca/etdhttps://ir.lib.uwo.ca/etd/6017. Accessed 14 Jun 2021.

20. Pećin I, Whittall R, Futema M, Sertić J, Reiner Ž, Leigh SEA, et al. Mutation detection in Croatian patients with Familial Hypercholesterolemia. Ann Hum Genet. 2013;77:22-30. doi:10.1111/j.1469-1809.2012.00735.x.

21. Sharifi $M$, Walus-Miarka $M$, Idzior-Waluś $B$, Malecki MT, Sanak $M$, Whittall R, et al. The genetic spectrum of familial hypercholesterolemia in south-eastern Poland. Metabolism. 2016;65:48-53. doi:10.1016/j. metabol.2015.10.018.

22. Hori M, Ohta N, Takahashi A, Masuda H, Isoda R, Yamamoto S, et al. Impact of LDLR and PCSK9 pathogenic variants in Japanese heterozygous familial hypercholesterolemia patients. Atherosclerosis. 2019;289:101-8. doi:10.1016/j.atherosclerosis.2019.08.004.
23. Martín-Campos JM, Plana N, Fiqueras R, Ibarretxe D, Caixàs A, Esteve $\mathrm{E}$, et al. Autosomal dominant hypercholesterolemia in Catalonia: Correspondence between clinical-biochemical and genetic diagnostics in 967 patients studied in a multicenter clinical setting. J Clin Lipidol. 2018;12:1452-62. doi:10.1016/j.jacl.2018.09.002.

24. Khera A V., Hegele RA. What Is Familial Hypercholesterolemia, and Why Does It Matter? Circulation. 2020;141:1760-3. doi:10.1161/ CIRCULATIONAHA.120.046961.

25. Chaudhary R, Garg J, Shah N, Sumner A. PCSK9 inhibitors: A new era of lipid lowering therapy. World J Cardiol. 2017;9:76. doi:10.4330/wjc. v9.i2.76.

26. Ajufo E, deGoma EM, Raper A, Yu KD, Cuchel M, Rader DJ. A randomized controlled trial of genetic testing and cascade screening in familial hypercholesterolemia. Genet Med. 2021;:1-8. doi:10.1038/ s41436-021-01192-z. 